\title{
Identification and frequency of the rs12516 and rs8176318 BRCA1 gene polymorphisms among different populations
}

\author{
FANG YANG, FENGXIA CHEN, JIN XU and XIAOXIANG GUAN
}

Department of Medical Oncology, Jinling Hospital, Medical School of Nanjing University, Nanjing, Jiangsu 210002, P.R. China

Received December 28, 2014; Accepted January 21, 2016

DOI: $10.3892 / 01.2016 .4252$

\begin{abstract}
Genetic mutation of breast cancer 1 (BRCAl) is one of the most notable factors responsible for a proportion of breast cancer cases. BRCAl encodes a 1,863-amino acid protein and functions as a negative regulator of tumor growth. Thus, investigation of the underlying mechanisms that regulate $B R C A 1$ gene expression provide further insight into possible targets for breast cancer therapy. Previous studies have demonstrated that the genetic variants in the BRCAl $3^{\prime}$ untranslated region (3'UTR), in addition to the cytosine-phosphate-guanine $(\mathrm{CpG})$ islands in the promoter region, are significantly associated with breast cancer risk; however, the role of single nucleotide polymorphisms (SNPs) in the BRCA1 3'UTR remains unclear. The present study aimed to investigate the association between SNPs and BRCAl mRNA expression levels. Bioinformatics analysis demonstrated that 2 SNPs in the BRCA1 3'UTR (rs12516 and rs8176318 with putative microRNA binding sites) were significantly correlated with mRNA expression in lymphoblastoid cell lines $\left(\mathrm{P}=2.55 \times 10^{-4}\right.$ and $\mathrm{P}=8.78 \times 10^{-5}$, respectively). Furthermore, the genotype frequency distribution varied between populations worldwide. In addition, $3 \mathrm{CpG}$ islands and several transcription factor binding sites in the $B R C A 1$ promoter region were established. The identification of such polymorphisms and $\mathrm{CpG}$ islands may aid in designing improved therapeutic strategies to treat patients with $B R C A 1$-associated breast cancer.
\end{abstract}

\section{Introduction}

According to the current literature, breast cancer is the most commonly diagnosed form of cancer and is the principal cause of cancer-associated fatality among females. The disease accounts for $14 \%$ of all cancer mortalities, particularly in

Correspondence to: Dr Xiaoxiang Guan, Department of Medical Oncology, Jinling Hospital, Medical School of Nanjing University, 305 East Zhongshan Road, Nanjing, Jiangsu 210002, P.R. China E-mail: xguan@nju.edu.cn

Key words: BRCA1, genetic polymorphism, $\mathrm{CpG}$ islands, breast cancer economically-developing countries, and $23 \%$ of all cancer cases (1). The breast cancer 1 (BRCA1) gene is $\sim 100 \mathrm{~kb}$ in length and is localized to chromosome 17q21; it is composed of 24 exons, with 22 of these translating into a 1,863 amino acid protein. It is currently well-established that mutations in the $B R C A 1$ gene result in a significantly increased lifetime risk for the development of breast cancer (2). Thus, BRCAl, functioning as a tumor suppressor, may be regarded as a strong candidate gene for breast cancer susceptibility.

The abnormal methylation of cytosine-phosphate-guanine $(\mathrm{CpG})$ islands in gene promoter regions is the predominant epigenetic mechanism by which gene transcription is effectively silenced; therefore, the transcriptional activity of genes with $\mathrm{CpG}$ island promoters is suppressed upon methylation (3). Notably, a previous study concluded that microRNAs (miRNAs/miRs) may function as oncogenes and tumor suppressors depending on the genetic variants in the 3'UTR binding sites, regulating gene expression post-transcriptionally (4). For example, miR-146a was observed to bind to the 3'UTR of BRCA1 and BRCA2 mRNA, potentially modulating their mRNA expression, and a genetic polymorphism in the miR-146a gene (rs2910164) was associated with a young diagnostic age of familial ovarian and breast cancer (5). However, the function of genetic variants in miRNA binding sites of $B R C A 1$ remains unclear. In the current study, the hypothesis that the BRCA1 3'UTR variants are associated with its mRNA expression was tested by performing bioinformatics analysis. In addition, the $\mathrm{CpG}$ islands and transcription factor binding sites (TFBSs) were predicted.

\section{Materials and methods}

Prediction of the CpG islands and TFBSs in the promoter region. The human $B R C A 1$ promoter region was obtained by searching the UCSC Genome Bioinformatics online database (www.genome.ucsc.edu/). The core promoters were identified by Neural Network Promoter Prediction (NNPP; www.fruitfly.org/seq_tools/promoter.html) and Promoter2.0 (www.cbs.dtu.dk/services/promoter/), and the $\mathrm{CpG}$ islands were predicted using the bioinformatics tool MethPrimer (www.urogene.org/methprimer/). The criteria used to define a $\mathrm{CpG}$ island was as follows: Island size, $>100$ bp; GC percentage, $>50.0$; and observed/expected ratio, $>0.6$. In addition, the TFBSs were predicted by JASPAR (jaspar.genereg.net/). 
Selection of polymorphisms in the 3'UTR and analysis of the genotype-phenotype association. The SNPs in the human BRCAl 3'UTR were identified using the National Center for Biotechnology Information SNP database (www.ncbi.nlm.nih.gov/SNP/). Subsequently, the distribution of all $B R C A 1$ genotypes among 11 distinctive populations was calculated. The 11 populations studied were as follows: Utah residents with Northern and Western European ancestry from the Center for the Study of Human Polymorphisms collection (CEU); Japanese individuals in Tokyo, Japan (JPT); members of the Yoruba tribe in Ibadan, Nigeria (YRI); Han Chinese individuals in Beijing, China (CHB); members of the Luhya tribe in Webuye, Kenya (LWK); individuals of Mexican ancestry in Los Angeles, California (MEX); Gujarati Indians in Houston, Texas (GIH); Chinese individuals in Metropolitan Denver, Colorado (CHD); individuals of African ancestry in Southwest USA (ASW); members of the Maasai tribe in Kinyawa, Kenya (MKK); and Tuscan individuals in Italy (TSI). SNP Function Prediction (snpinfo.niehs.nih. gov/snpinfo/snpfunc.htm) was utilized to predict the possible miRNA binding sites in the BRCAl gene. Additionally, SNPs not in linkage disequilibrium $\left(\mathrm{LD} ; \mathrm{r}^{2}<0.8\right)$ were selected and LD maps of these SNPs in the BRCAl gene were plotted using the online program (snpinfo.niehs.nih.gov/snpinfo/snptag.htm). Additional data was utilized regarding BRCAl genotypes and mRNA levels, which was available from the HapMap database (http://app3.titan.uio.no/biotools/tool.php?app=snpexp), for the genotype-phenotype association analysis (6). The genotyping data from the HapMap phase II release 23 dataset consists of $\sim 4$ million SNP genotypes from 270 individuals belonging to 4 different populations (7).

Statistical analysis. The genotype and phenotype correlation was analyzed using the $\chi^{2}$ test. Statistical analysis was performed on SPSS version 21 software (IMB SPSS, Armonk, NY, USA) All statistical tests were two-sided, and $\mathrm{P}<0.05$ was considered to indicate a statistically significance difference.

\section{Results}

$C p G$ islands and TFBSs in the BRCA1 promoter region. The online tools NNPP and Promoter2.0 indicated that there were several core promoter regions located in the target sequence. A total of 3 core promoter regions were determined by NNPP, whilst Promoter 2.0 identified 2 core promoter regions. The bioinformatics software MethPrimer was used to predict the range of the $\mathrm{CpG}$ islands in the $B R C A 1$ gene. The results determined that there were $3 \mathrm{CpG}$ islands in the $B R C A \mathrm{I}$ promoter region (Fig. 1; Table I), which may serve a crucial role in the expression of the BRCA1 gene. In total, 31 transcription factors and 45 binding sites were predicted in the target sequence regions when limiting the relative profile score threshold to $>95 \%$.

BRCA1 3'UTR selected variants and their putative miRNA binding sites. A total of 28 SNPs were identified in the human BRCA1 3'UTR; 13 of these SNPs had available minor allele frequency (MAF) values, 2 of which (rs12516 and rs8176318) had MAF values $>0.05$. SNP Function Prediction demonstrated that these 2 SNPs exhibited putative miRNA binding
Table I. CpG island prediction results.

\begin{tabular}{lccr}
\hline CpG island & Size, bp & Start & End \\
\hline 1 & 129 & 412 & 540 \\
2 & 244 & 608 & 851 \\
3 & 221 & 950 & 1170 \\
\hline
\end{tabular}

Criteria used were as follows: Island size, $>100 \mathrm{bp}$; $\mathrm{GC}$ percent, $>50.0$; observed to expected ratio, $>0.6$. CpG, cytosine-phosphate-guanine.

sites (Table II). As presented in Table II, the C-to-thymine (T) transition mutation, rs12516, had 6 potential miRNA binding sites, namely, hsa-miR-188-5p, hsa-miR-502-5p, hsa-miR-557, hsa-miR-623, hsa-miR-637 and hsa-miR-639. The G-to-T transition mutation, rs8176318, was observed to share hsa-miR-639 with rs12516, and contain 4 other miRNA binding sites, namely, hsa-miR-1182, hsa-miR-149, hsa-miR-345 and hsa-miR-544.

Frequency distribution of selected variants among distinct populations. As previously reported, the derived alleles at rs12516 and rs8176318 in the BRCAI $3^{\prime}$ UTR demonstrated a positive association with familial ovarian and breast cancer in Thai women, and the 2 SNPs were in strong linkage disequilibrium in populations and varied by ethnicity (8). In the present study, in order to better evaluate the global genotypes of rs12516 and rs8176318 in BRCAl, the frequency distribution data of these variants across 11 worldwide populations were summarized (Tables III and IV). The 2 SNPs presented differences in genotype frequency distribution among the worldwide populations. For rs12516, the genotype frequencies of CC, CT and TT were highest in YRI (69.0\%), CHD (57.6\%) and GIH (18.2\%). The frequency distributions of the GG, GT and TT genotypes of rs8176318 ranked first among LWK (84.4\%), CHD (56.6\%) and GIH (18.2\%). The T allele frequencies of rs12516 in the various populations ranged between $44.9 \%$ in GIH and $17.3 \%$ in YRI. Notably, the frequency distributions of the GG, GT and TT genotypes of rs8176318 were the same as the data for CC, CT and TT genotypes of rs12516 among the CEU and CHB populations.

LD of all SNPs in the BRCA1 gene calculation. SNPs not in $\mathrm{LD}\left(\mathrm{r}^{2}<0.8\right)$ were selected and LD maps of those SNPs in the $B R C A 1$ gene were plotted using the SNP Function Prediction program to identify the potential functional relevance of all selected SNPs (Fig. 2). The degree of pairwise LD between all SNPs was estimated as quantified by the disequilibrium coefficient $\left(D^{\prime}\right)$ and $r^{2}$, which represented the proportion of disequilibrium and the maximum possible disequilibrium given for observed allele frequencies, respectively. The higher the $\mathrm{D}^{\prime}$ value, the greater the association between the two loci being studied. The color of each SNP spot reflects its $\mathrm{D}^{\prime}$ value, which changes from red to white as it decreases. The MAF of each aforementioned allele was $>0.05$. As for the SNPs in the BRCAl 3'UTR, only rs12516 and rs8176318 were included in the LD plot, but neither were the predicted tag SNP. 
Table II. Selected SNPs of 3'UTR and putative miRNA binding sites.

\begin{tabular}{|c|c|c|c|}
\hline SNP & Alleles & MAF, $\%$ & Putative miRNA binding sites \\
\hline rs8176320 & $\mathrm{G}>\mathrm{A}$ & 0.51 & $\begin{array}{l}\text { hsa-miR-101, hsa-miR-15a, hsa-miR-15b, } \\
\text { has-miR-16, hsa-miR-194, hsa-miR-195, } \\
\text { hsa-miR-424, hsa-miR-450b-5p, hsa-miR-545 }\end{array}$ \\
\hline rs184237074 & $\mathrm{C}>\mathrm{T}$ & 0.05 & NA \\
\hline rs189382442 & $\mathrm{T}>\mathrm{C}$ & 0.05 & NA \\
\hline rs182218567 & $\mathrm{A}>\mathrm{G}$ & 0.05 & NA \\
\hline rs12516 & $\mathrm{C}>\mathrm{T}$ & 31.18 & $\begin{array}{l}\text { hsa-miR-188-5p, hsa-miR-502-5p, hsa-miR-557, } \\
\text { hsa-miR-623, hsa-miR-637, hsa-miR-639 }\end{array}$ \\
\hline rs111791349 & $\mathrm{C}>\mathrm{T}$ & 0.51 & NA \\
\hline rs185966495 & $\mathrm{G}>\mathrm{C}$ & 0.05 & NA \\
\hline rs8176319 & $\mathrm{C}>\mathrm{T}$ & 0.69 & NA \\
\hline rs138782023 & $\mathrm{T}>\mathrm{C}$ & 0.28 & NA \\
\hline rs141850147 & $\mathrm{G}>\mathrm{A}$ & 0.05 & NA \\
\hline rs8176318 & $\mathrm{G}>\mathrm{T}$ & 29.06 & $\begin{array}{l}\text { hsa-miR-1182, hsa-miR-149, hsa-miR-345, } \\
\text { hsa-miR-544, hsa-miR-639 }\end{array}$ \\
\hline rs56108540 & $\mathrm{T}>\mathrm{C}$ & 0.23 & $\begin{array}{c}\text { hsa-miR-125a-3p, hsa-miR-224, hsa-miR-499-3p } \\
\text { hsa-miR-539, hsa-miR-548c-3p } \\
\text { hsa-miR-767-5p }\end{array}$ \\
\hline rs137892861 & $\mathrm{G}>\mathrm{A}$ & 0.14 & NA \\
\hline
\end{tabular}

SNP, single nucleotide polymorphism; 3'UTR, 3' untranslated region; miRNA/miR, microRNA; G, guanine; A, adenine; C, cytosine; T, thymine; MAF, minor allele frequency; hsa, Homo sapiens; NA, not available.

Table III. Genotype frequency of the breast cancer 1 gene rs 12516 polymorphism in different populations.

\begin{tabular}{|c|c|c|c|c|c|c|c|}
\hline \multirow[b]{2}{*}{ Populations } & \multirow[b]{2}{*}{$\mathrm{n}$} & \multicolumn{3}{|c|}{ Genotype frequency, n (\%) } & \multicolumn{2}{|c|}{ Allele frequency, $\%$} & \multirow[b]{2}{*}{ HWP } \\
\hline & & $\mathrm{CC}$ & $\mathrm{CT}$ & $\mathrm{TT}$ & $\mathrm{C}$ & $\mathrm{T}$ & \\
\hline CEU & 226 & $100(44.2)$ & $102(45.1)$ & $24(10.6)$ & 66.8 & 33.2 & 1.000 \\
\hline JPT & 170 & $82(48.2)$ & $76(44.7)$ & $12(7.1)$ & 70.6 & 29.4 & 0.527 \\
\hline YRI & 226 & $156(69.0)$ & $62(27.4)$ & $8(3.5)$ & 82.7 & 17.3 & 0.752 \\
\hline $\mathrm{CHB}$ & 82 & $38(46.3)$ & $32(39.0)$ & $12(14.6)$ & 65.9 & 34.1 & 0.403 \\
\hline LWK & 180 & $112(62.2)$ & $62(34.4)$ & $6(3.3)$ & 79.4 & 20.6 & 0.655 \\
\hline MEX & 98 & $62(63.3)$ & $26(26.5)$ & $10(10.2)$ & 76.5 & 23.5 & 0.100 \\
\hline GIH & 176 & $50(28.4)$ & $94(53.4)$ & $32(18.2)$ & 55.1 & 44.9 & 0.479 \\
\hline CHD & 170 & $46(27.1)$ & $98(57.6)$ & $26(15.3)$ & 55.9 & 44.1 & 0.150 \\
\hline ASW & 98 & $44(44.9)$ & $50(51.0)$ & $4(4.1)$ & 70.4 & 29.6 & 0.150 \\
\hline MKK & 286 & $172(60.1)$ & $104(36.4)$ & $10(3.5)$ & 78.3 & 21.7 & 0.403 \\
\hline TSI & 174 & $64(36.8)$ & $88(50.6)$ & $22(12.6)$ & 62.1 & 37.9 & 0.527 \\
\hline
\end{tabular}

CEU, Utah residents with Northern and Western European ancestry from the Center for the Study of Human Polymorphisms collection; JPT, Japanese individuals in Tokyo, Japan; YRI, members of the Yoruba tribe in Ibadan, Nigeria; CHB, Han Chinese individuals in Beijing, China; LWK, members of the Luhya tribe in Webuye, Kenya; MEX, individuals of Mexican ancestry in Los Angeles, California; GIH, Gujarati Indians in Houston, Texas; CHD, Chinese individuals in Metropolitan Denver, Colorado; ASW, individuals of African ancestry in Southwest, USA; MKK, members of the Maasai tribe in Kinyawa, Kenya; TSI, Tuscan individuals in Italy; HWP, Hardy-Weinburg probability; C, cytosine; T, thymine.

BRCA1 mRNA expression by genotypes in lymphoblastoid cell lines. The present study took advantage of the available HapMap-complementary DNA expression database for the correlation analysis between $B R C A 1$ genotype and mRNA expression in 270 lymphoblastoid cell lines. Excluding the 6 cell lines with unavailable values for rs12516, 20 (7.6\%) cell lines exhibited the TT genotype, 108 (40.9\%) cell lines exhibited the CT genotype and $136(51.5 \%)$ cell lines exhibited 
Table IV. Genotype frequency of the breast cancer 1 gene rs8176318 polymorphism in different populations.

\begin{tabular}{|c|c|c|c|c|c|c|c|}
\hline \multirow[b]{2}{*}{ Populations } & \multirow[b]{2}{*}{$\mathrm{n}$} & \multicolumn{3}{|c|}{ Genotype frequency, n (\%) } & \multicolumn{2}{|c|}{ Allele frequency, \% } & \multirow[b]{2}{*}{ HWP } \\
\hline & & GG & GT & $\mathrm{TT}$ & $\mathrm{G}$ & $\mathrm{T}$ & \\
\hline CEU & 226 & $100(44.2)$ & $102(45.1)$ & $24(10.6)$ & 66.8 & 33.2 & 1.000 \\
\hline JPT & 172 & $88(51.2)$ & 72 (41.9) & $12(7.0)$ & 72.1 & 27.9 & 0.752 \\
\hline YRI & 226 & $180(79.6)$ & 42 (18.6) & $4(1.8)$ & 88.9 & 11.1 & 0.584 \\
\hline $\mathrm{CHB}$ & 82 & $38(46.3)$ & $32(39.0)$ & $12(14.6)$ & 65.9 & 34.1 & 0.403 \\
\hline LWK & 180 & $152(84.4)$ & $26(14.4)$ & $2(1.1)$ & 91.7 & 08.3 & 0.655 \\
\hline MEX & 98 & $62(63.3)$ & $28(28.6)$ & $8(8.2)$ & 77.6 & 22.4 & 0.251 \\
\hline GIH & 176 & $52(29.5)$ & $92(52.3)$ & $32(18.2)$ & 55.7 & 44.3 & 0.584 \\
\hline CHD & 170 & $48(28.2)$ & $96(56.5)$ & $26(15.3)$ & 56.5 & 43.5 & 0.200 \\
\hline ASW & 98 & $56(57.1)$ & 42 (42.9) & $0(0)$ & 78.6 & 21.4 & 0.251 \\
\hline MKK & 286 & $206(72.0)$ & $72(25.2)$ & $8(2.8)$ & 84.6 & 15.4 & 0.752 \\
\hline TSI & 176 & $68(38.6)$ & $88(50.0)$ & $20(11.4)$ & 63.6 & 36.4 & 0.479 \\
\hline
\end{tabular}

CEU, Utah residents with Northern and Western European ancestry from the Center for the Study of Human Polymorphisms collection; JPT, Japanese individuals in Tokyo, Japan; YRI, members of the Yoruba tribe in Ibadan, Nigeria; CHB, Han Chinese individuals in Beijing, China; LWK, members of the Luhya tribe in Webuye, Kenya; MEX, individuals of Mexican ancestry in Los Angeles, California; GIH, Gujarati Indians in Houston, Texas; CHD, Chinese individuals in Metropolitan Denver, Colorado; ASW, individuals of African ancestry in Southwest, USA; MKK, members of the Maasai tribe in Kinyawa, Kenya; TSI, Tuscan individuals in Italy; HWP, Hardy-Weinburg probability; C, cytosine; T, thymine; G, guanine.

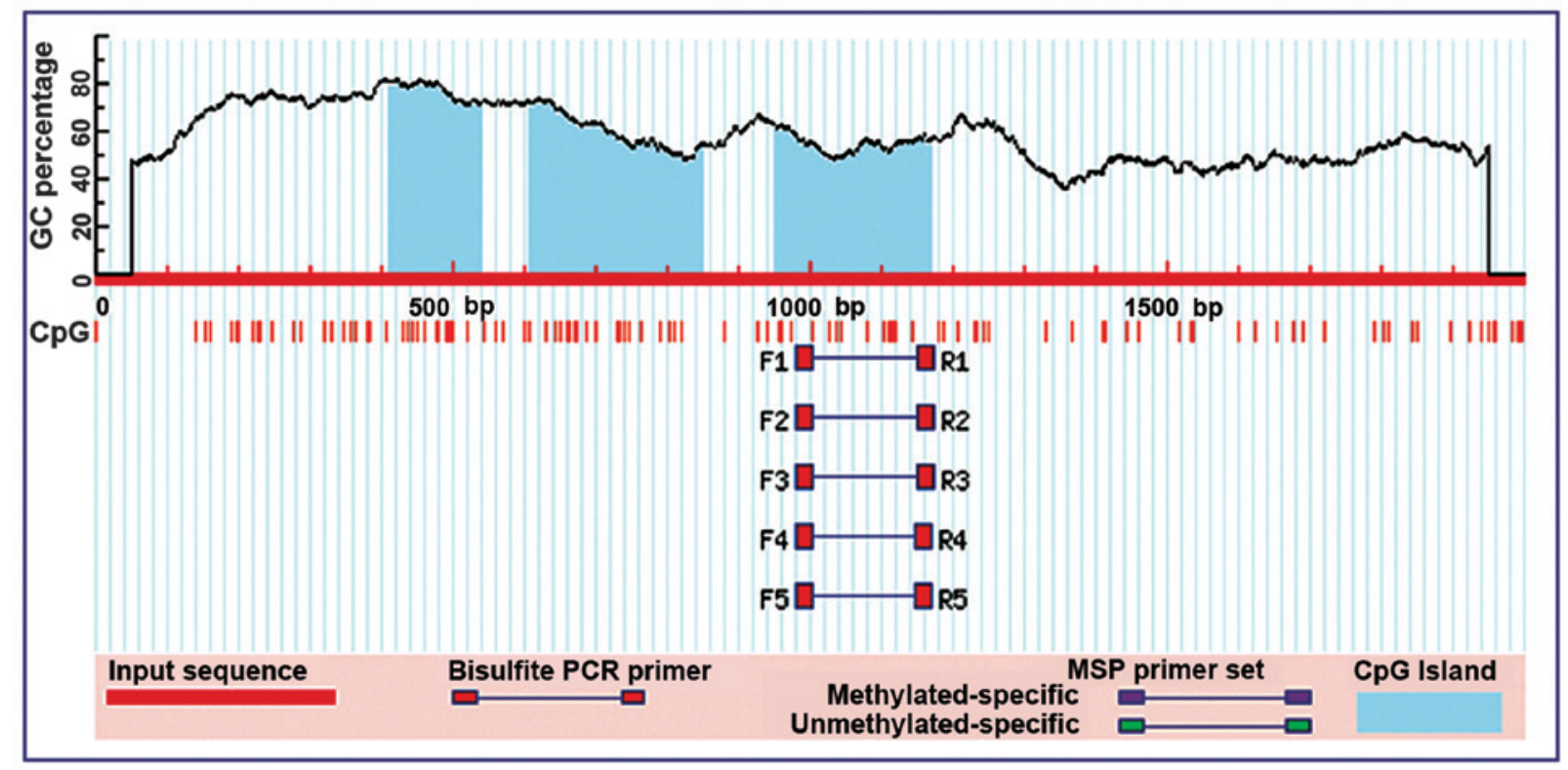

Figure 1. $\mathrm{CpG}$ island prediction results in the breast cancer 1 gene promoter region. Criteria used to define a $\mathrm{CpG}$ island were as follows: Island size, $>100$; $\mathrm{GC}$ percentage, $>50.0$; and observed/expected ratio, $>0.6$. CpG, cytosine-phosphate-guanine; PCR, polymerase chain reaction; MSP, methylation-specific PCR.

the CC genotype. For rs8176318, 19 (7.0\%) cell lines exhibited the TT genotype, 95 (35.2\%) exhibited the GT genotype and $156(57.8 \%)$ exhibited the GG genotype. The effect of the rs12516 and rs8176318 genotypes on BRCA1 mRNA expression levels is presented in Fig. 3. For rs12516, the BRCAI mRNA expression level was significantly decreased in the CC genotype compared with the CT and TT genotypes $\left(\mathrm{P}=2.55 \times 10^{-4}\right.$; Fig. 3A). Similarly, the rs8176318 GG genotype had a significantly lower expression level than the GT and TT genotypes ( $\mathrm{P}=8.78 \times 10^{-5}$; Fig. 3B).

\section{Discussion}

Since the identification of the BRCAl gene 20 years ago (9), the biological basis underlying the high breast cancer risk in women with BRCAl mutations has gained increasing attention. $B R C A 1$ operates in a series of cellular processes, including DNA repair, chromatin remodeling, protein ubiquitination, regulation of transcription, apoptosis and cell cycle checkpoint control. As observed in carriers of germline BRCAl mutations, the disruption of any of the aforementioned processes may result 


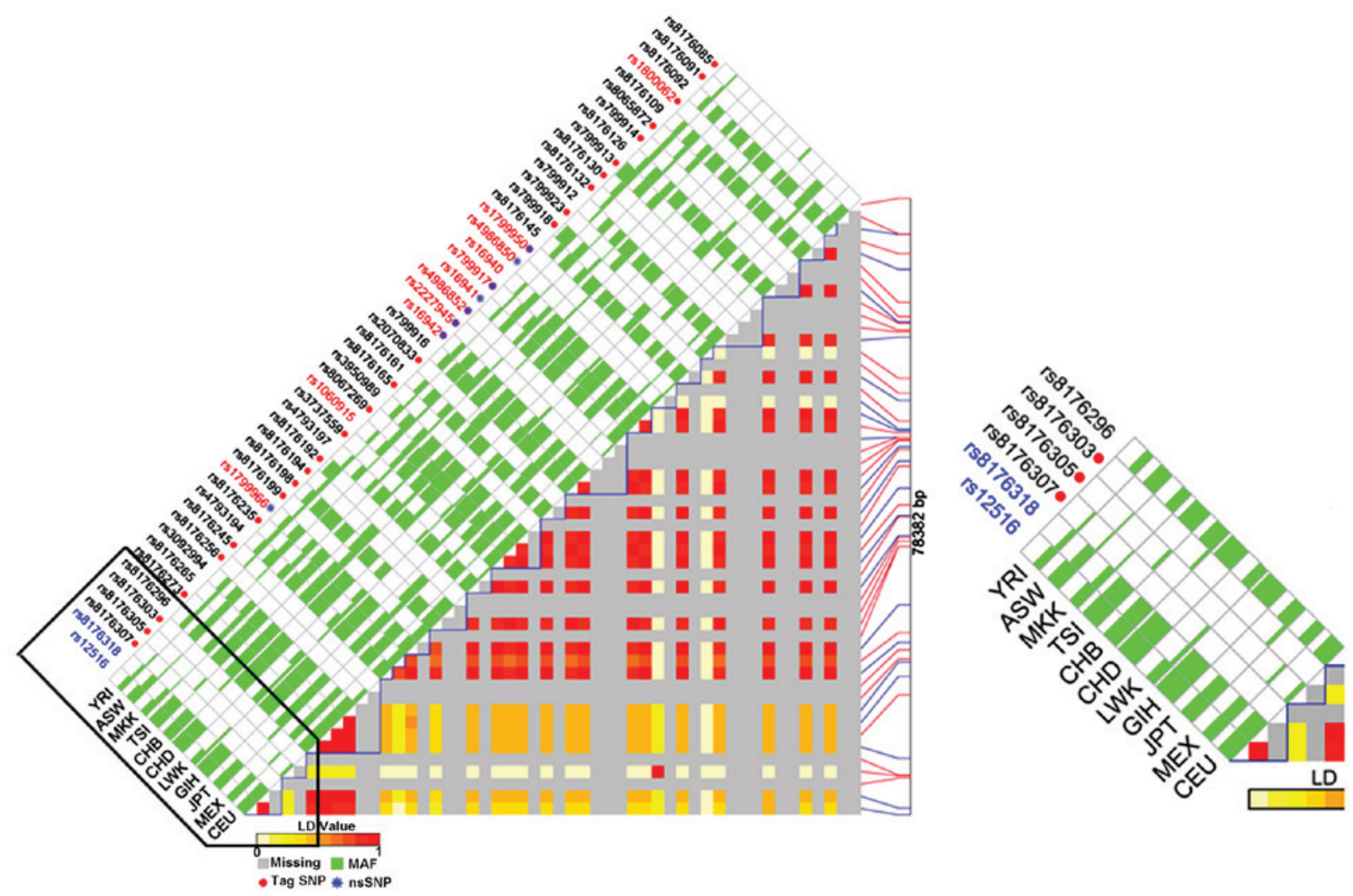

Figure 2. Linkage disequilibrium plot of the breast cancer 1 gene region using SNP function prediction. The color of each SNP spot reflects its D' value, which changes from red to white as the D' value decreases. SNP, single nucleotide polymorphism; LWK, members of the Luhya tribe in Webuye, Kenya; CHD, Chinese individuals in Metropolitan Denver, Colorado; YRI, members of the Yoruba tribe in Ibadan, Nigeria; MEX, individuals of Mexican ancestry in Los Angeles, California; JPT, Japanese individuals in Tokyo, Japan; GIH, Gujarati Indians in Houston, Texas; CHB, Han Chinese individuals in Beijing, China; CEU, Utah residents with Northern and Western European ancestry from the Center for the Study of Human Polymorphismscollection; LD, linkage disequilibrium; MAF, minor allele frequency; nsSNP, non-synonymous SNP.

A

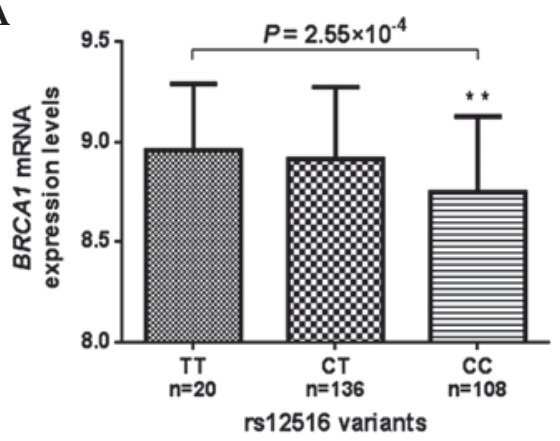

B

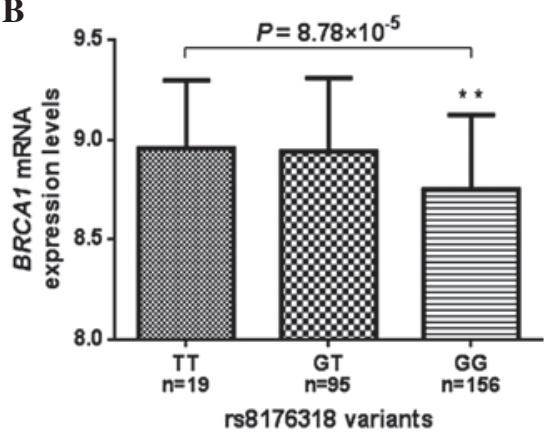

Figure 3. Genotype-phenotype association analysis of the mRNA expression level of the BRCA1 variants. (A) rs12516 and (B) rs8176318 genotypes and mRNA expression in Epstein-Barr virus-transformed lymphoblastoid cell lines from the HapMap database. ${ }^{* *} \mathrm{P}<0.001$. BRCA1, breast cancer 1; C, cytosine; T, thymine; G, guanine.

in an increased risk for carcinogenesis (10). Dacheux et al (11) demonstrated that $B R C A l$ modified the translational regulation of $\sim 7 \%$ of genes expressed in MCF7 cells, including structural maintenance of chromosomes 6 , thyroid hormone receptor $\alpha$ and topoisomerase I. It was proposed that $B R C A 1$ may serve a direct transcriptional role in the regulation of $\mathrm{p} 27^{\mathrm{Kip1}}$ resulting in S-phase arrest; this suggested that the BRCAl-mediated transcriptional regulation of $\mathrm{p} 27^{\mathrm{Kip} 1}$ may function as a mechanism for $B R C A 1$-induced growth inhibition (12).

$B R C A 1$ promoter methylation is considered to serve a key role in the etiology of human breast cancer. Iwamoto et al (13) reported that $B R C A 1$ promoter methylation in peripheral blood cells may establish a novel risk factor for the development of breast cancer. Xu et al (14) demonstrated that methylation of the $B R C A 1$ promoter was associated with increased mortality rates among women with breast cancer. The promoter region of the BRCAl gene was methylated in a large proportion of Taiwanese patients with early-stage breast cancer, and patients with $B R C A$ l-methylated tumors exhibited poorer survival outcomes (15). A meta-analysis provided evidence that methylation of the BRCAl promoter was associated with the poor survival of patients with breast cancer (16). To directly investigate 
BRCAl gene regulation on a transcriptional level, the present study predicted $3 \mathrm{CpG}$ islands and several putative TFBSs in the $B R C A 1$ promoter region using bioinformatics analysis.

Previous studies have demonstrated that BRCAl 3'UTR miRNA binding site variants are associated with breast cancer risk post-translationally. For example, miR-24 was observed to directly target the 3'UTR of BRCAl and resulted in significant repression of the $B R C A l$ gene (17). In addition, it was demonstrated that the BRCAl polymorphism, rs799917, was associated with breast cancer risk (18). By contrast, Hasan et al (19) reported that rs799917 demonstrated no significant association with breast cancer in 100 patients with breast cancer in Saudi Arabia (19). Therefore, the role of genetic variants in the BRCA1 3'UTR and its post-transcriptional regulation remains unclear. Such differences may be due to reproductive patterns, in addition to exposure to particular environmental carcinogens, different lifestyles and different genetic backgrounds (19). The present study focused on the polymorphisms in the BRCA1 3'UTR, and identified that rs12516 and rs 8176318 had potential miRNA binding sites. Furthermore, rs12516 and rs8176318 exhibited differences in genotype frequency distribution among populations worldwide. Each SNP had a significant association with BRCAl mRNA expression, therefore implying that these SNPs may partially contribute to $B R C A 1$ post-transcriptional regulation.

In conclusion, the current study predicted $3 \mathrm{GpG}$ islands, 45 TFBSs located in the promoter region and 13 SNPs located in the 3'UTR of the BRCAl gene. A total of 4 SNPs (rs8176320, rs12516, rs8176318 and rs56108540) were confirmed to have putative miRNA binding sites, among which only 2 (rs12516 and rs8176318) had MAF values $>0.05$. These 2 SNPs, rs 12516 and rs8176318, each demonstrated a significant association with $B R C A 1 \mathrm{mRNA}$ expression. The results from the present study have raised the possibility that rs12516 and rs8176318 may be associated with an increased risk of breast cancer by altering the BRCAl mRNA level. However, such results require substantiation by experimental data, therefore further investigations are warranted to confirm the function of these polymorphisms. In addition, further functional analysis is necessary to validate the promoter $\mathrm{CpG}$ islands and SNPs in the 3'UTR to allow for investigation of $B R C A 1$ gene regulation as a potential therapy for breast cancer.

\section{Acknowledgements}

This study was supported by the Foundation for Clinical Medicine Science and Technology Special Project of the Jiangsu Province, China (grant no. BL2014071) to X.G.

\section{References}

1. Jemal A, Bray F, Center MM, Ferlay J, Ward E and Forman D: Global cancer statistics. CA Cancer J Clin 61: 69-90, 2011.
2. Ayoub N, Lucas C and Kaddoumi A: Genomics and pharmacogenomics of breast cancer: Current knowledge and trends. Asian Pac J Cancer Prev 12: 1127-1140, 2011.

3. Zeisberg EM and Zeisberg M: The role of promoter hypermethylation in fibroblast activation and fibrogenesis. J Pathol 229: 264-273, 2013.

4. Hook L, Hancock M, Landais I, Grabski R, Britt W and Nelson JA: Cytomegalovirus microRNAs. Curr Opin Virol 7: 40-46, 2014.

5. Shen J, Ambrosone CB, DiCioccio RA, Odunsi K, Lele SB and Zhao H: A functional polymorphism in the miR-146a gene and age of familial breast/ovarian cancer diagnosis. Carcinogenesis 29: 1963-1966, 2008.

6. Holm K, Melum E, Franke A and Karlsen TH: SNPexp - A web tool for calculating and visualizing correlation between HapMap genotypes and gene expression levels. BMC Bioinformatics 11: 600, 2010.

7. International HapMap Consortium: The International HapMap Project. Nature 426: 789-796, 2003.

8. Pelletier C, Speed WC, Paranjape T, Keane K, Blitzblau R, Hollestelle A, Safavi K, van den Ouweland A, Zelterman D, Slack FJ, et al: Rare BRCA1 haplotypes including 3'UTR SNPs associated with breast cancer risk. Cell Cycle 10: 90-99, 2011.

9. Narod SA, Feunteun J, Lynch HT, Watson P, Conway T, Lynch J and Lenoir GM: Familial breast-ovarian cancer locus on chromosome 17q12-q23. Lancet 338: 82-83, 1991.

10. Yang ES and Xia F: BRCA1 16 years later: DNA damage-induced BRCA1 shuttling. FEBS J 277: 3079-3085, 2010.

11. Dacheux E, Vincent A, Nazaret N, Combet C, Wierinckx A, Mazoyer S,DiazJJ,Lachuer J and Venezia ND: BRCA1-Dependent Translational Regulation in Breast Cancer Cells. PLoS One 8: e67313, 2013.

12. Williamson EA, Dadmanesh F and Koeffler HP: BRCA1 transactivates the cyclin-dependent kinase inhibitor p27(Kip1). Oncogene 21: 3199-3206, 2002.

13. Iwamoto T, Yamamoto N, Taguchi T, Tamaki Y and Noguchi S: BRCA1 promoter methylation in peripheral blood cells is associated with increased risk of breast cancer with BRCA1 promoter methylation. Breast Cancer Res Treat 129: 69-77, 2011.

14. Xu X, Gammon MD, Zhang Y, Bestor TH, Zeisel SH, Wetmur JG, Wallenstein S, Bradshaw PT, Garbowski G, Teitelbaum SL, et al: BRCA1 promoter methylation is associated with increased mortality among women with breast cancer. Breast Cancer Res Treat 115: 397-404, 2009.

15. Hsu NC, Huang YF, Yokoyama KK, Chu PY, Chen FM and Hou MF: Methylation of BRCA1 promoter region is associated with unfavorable prognosis in women with early-stage breast cancer. PLoS One 8: e56256, 2013.

16. Wu L, Wang F, Xu R, Zhang S, Peng X, Feng Y, Wang J and $\mathrm{Lu}$ C: Promoter methylation of BRCA1 in the prognosis of breast cancer: A meta-analysis. Breast Cancer Res Treat 142: 619-627, 2013.

17. Lal A, Navarro F, Maher CA, Maliszewski LE, Yan N, O'Day E, Chowdhury D, Dykxhoorn DM, Tsai P, Hofmann O, et al: miR-24 Inhibits cell proliferation by targeting E2F2, MYC and other cell-cycle genes via binding to 'seedless' 3'UTR microRNA recognition elements. Mol Cell 35: 610-625, 2009.

18. Huo X, Lu C, Huang X, Hu Z, Jin G, Ma H, Wang X, Qin J, Wang $X$, Shen $H$ and Tang J: Polymorphisms in BRCA1, BRCA1-interacting genes and susceptibility of breast cancer in Chinese women. J Cancer Res Clin Oncol 135: 1569-1575, 2009.

19. Hasan TN, Shafi G, Syed NA, Alsaif MA, Alsaif AA and Alshatwi AA: Lack of association of BRCA1 and BRCA2 variants with breast cancer in an ethnic population of Saudi Arabia, an emerging high-risk area. Asian Pac J Cancer Prev 14: 5671-5674, 2013 . 